\title{
Supporting Information for Studying and Switching Electron Transfer: From the Ensemble to the Single Molecule
}

Michael W. Holman, Ruchuan Liu, Ling Zang, ${ }^{\ddagger}$ Ping Yan, Sara A. DiBenedetto, Robert D. Bowers, David M. Adams*

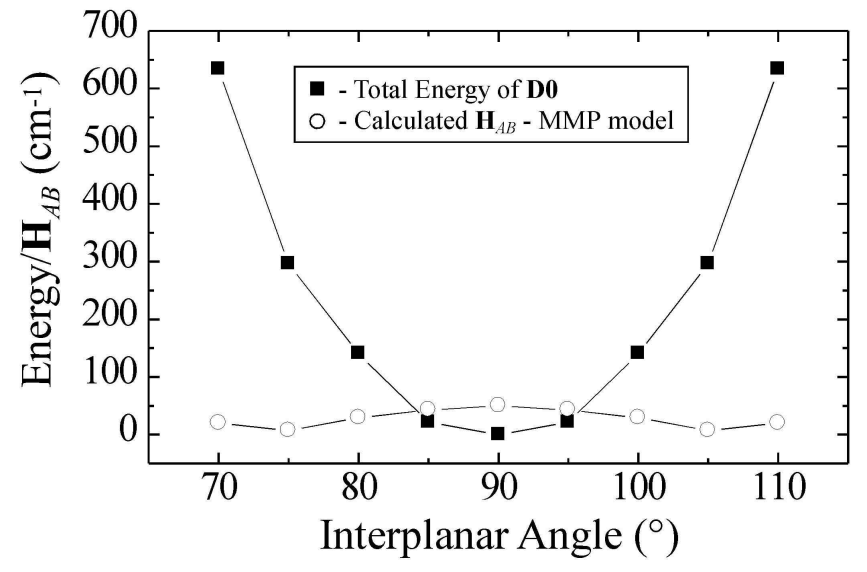

Figure S1. Rotational potential for D0 (using DFT at the B3LYP level and a 6-31G** basis set) and calculated rotational dependence of $\mathbf{H}_{A B}$ (using the MMP model, as described in the text) at the $\mathbf{D 0}$ distance $\left(1.38 \AA\right.$ ). The value for $\mathbf{H}_{A B}$ decreases slightly from $90^{\circ}$ to $75^{\circ}$, before increase to a maximum at $0^{\circ}$. 